\title{
Comparison of demographics, cardiovascular risk factors profile and prevalence of coexistent atherosclerotic vascular disease in patients with severe aortic stenosis stratified according to dichotomized stenosis severity
}

\author{
Beata Bobrowska ${ }^{1,2}$, Wojciech Zasada ${ }^{2}$, Artur Dziewierz ${ }^{1,2}$, Olga Kruszelnicka ${ }^{3, *}$, Andrzej Surdacki ${ }^{1,2, *}$, \\ Dariusz Dudek ${ }^{1,2, *}$
}

${ }^{1}$ Second Department of Cardiology, Jagiellonian University Medical College, Krakow, Poland ${ }^{2}$ Second Department of Cardiology and Cardiovascular Interventions, University Hospital, Krakow, Poland ${ }^{3}$ Department of Coronary Artery Disease and Heart Failure, John Paul II Hospital, Krakow, Poland

*Joint senior authors on this work.

Adv Interv Cardiol 2017; 13, 4 (50): 331-334 DOI: https://doi.org/10.5114/aic.2017.71616

\section{Introduction}

Degenerative calcific aortic stenosis (AS) is the most frequent valvular heart disease in Europe and North America. The prevalence of this AS form is especially pronounced in the elderly, representing a major public health issue [1-5]. The choice of optimal management of AS patients requires appropriate identification of subjects with severe AS, and echocardiography is a first-line method to diagnose AS and estimate its severity [5]. According to clinical practice guidelines, echocardiographic criteria of severe AS include a calculated aortic valve area (AVA) $<1.0 \mathrm{~cm}^{2}$, mean transaortic valve pressure gradient $>40 \mathrm{~mm} \mathrm{Hg}$ or maximal flow velocity > $4 \mathrm{~m} / \mathrm{s}$ [5]. Inconsistencies in AS grading were confirmed by cardiac catheterization and a calculated AVA of $0.8 \mathrm{~cm}^{2}$ corresponded to a mean transvalvular pressure gradient of $40 \mathrm{~mm} \mathrm{Hg}$ by echocardiography in patients with severe AS and a normal left ventricular stroke volume [6].

\section{Aim}

Our aim was to compare clinical characteristics of patients with severe degenerative AS according to the degree of AVA narrowing categorized by an AVA cut-off value of $0.8 \mathrm{~cm}^{2}$.

\section{Material and methods}

We retrospectively analyzed data of 145 previously described [7] consecutive patients (66 women and 79 men) with severe degenerative AS who were admitted to our ter- tiary center between January 2003 and October 2012. The diagnosis of severe AS was based on recognized echocardiographic criteria [5]. All examinations were performed by one of two well-experienced sonographers with the highest level of competence in our department. All patients underwent elective coronary angiography and carotid ultrasonography as a part of routine diagnostic work-up. Patients' characteristics obtained during the index hospitalization were recorded. The ethics committee of our university was notified about the registry and no objection was raised. A detailed study design was published previously, as well as results of a data analysis primarily focused on the determinants of coexistent coronary and carotid atherosclerosis [7].

Cardiovascular risk factors (arterial hypertension, hypercholesterolemia, diabetes mellitus and self-reported current smoking habit) were defined according to current recommendations as reported previously [7]. Estimated glomerular filtration rate (eGFR) was calculated according to the simplified Modification of Diet in Renal Disease Study formula. Significant coronary artery disease (CAD) was defined as a history of coronary revascularization or the presence of $\geq 1$ diameter stenosis of $\geq 50 \%$ of at least one major epicardial coronary artery on coronary angiography [7]. Relevant internal carotid artery stenosis (ICAS) was defined as stenosis of $\geq 50 \%$ of at least one internal carotid artery [7].

\section{Statistical analysis}

Data are shown as mean and standard deviation (SD) for continuous characteristics and numbers $(n)$ and per- 
centages for categorical variables. The patients were divided into two groups according to the AVA value: group A with an AVA in the range $0.8-1.0 \mathrm{~cm}^{2}$, and group $B$ with an AVA $<0.8 \mathrm{~cm}^{2}$. Intergroup differences were calculated by a two-sided Student's $t$ test for continuous data and two-tailed Fisher's exact test for proportions.

In order to identify independent predictors of categorized stenosis severity, multiple logistic regression was performed with a dichotomized AVA $\left(<0.8 \mathrm{~cm}^{2}\right.$ vs. $0.8-1.0 \mathrm{~cm}^{2}$ ) as a dependent variable, and age, gender, eGFR and the number of selected traditional risk factors (hypertension, hypercholesterolemia, diabetes mellitus and current smoking habit) as potential predictors. The Hosmer-Lemeshow test was performed to verify the goodness of fit of the regression equation.

In addition, because we had previously described an effect of the age-gender interaction on the prevalence of significant CAD [7], we evaluated whether a similar interaction might affect categorized AS severity. Accord- ingly, we created an interaction term which was set to 1 in women older than 76 years, and equalled 0 in the remainder. Then the significance of the possible age-gender interaction was tested by entering the interaction term, gender and categorized age (according to a median of 76 years) into a supplemental logistic regression analysis with a dichotomized AVA as a dependent variable.

\section{Statistical analysis}

A $p$-value below 0.05 was assumed significant. All analyses were performed using a licensed statistical software: Statistica (data analysis software system), version 12 (StatSoft, Inc., 2014, Tulsa, OK, USA).

\section{Results}

As shown in Table I, patients with more advanced AS (group B: AVA $<0.8 \mathrm{~cm}^{2}$ ) were older, more frequently women, and with a lower prevalence of smoking habit compared to subjects with an AVA in the range

Table I. Patients' characteristics according to dichotomized aortic valve area (AVA)

\begin{tabular}{|c|c|c|c|}
\hline Characteristics & $\begin{array}{c}\text { Group A } \\
\text { AV: } 0.8-1.0 \mathrm{~cm}^{2} \\
N=67\end{array}$ & $\begin{array}{c}\text { Group B } \\
\text { AVA }<0.8 \mathrm{~cm}^{2} \\
N=78\end{array}$ & $P$-value \\
\hline \multicolumn{4}{|l|}{ Age and gender: } \\
\hline Age [years] & $72(9)$ & $77(9)$ & 0.006 \\
\hline Gender, men/women, $n$ (\%) & $44 / 23(66 / 34)$ & $35 / 43(45 / 55)$ & 0.013 \\
\hline \multicolumn{4}{|l|}{ Clinical characteristics, $n(\%)$ : } \\
\hline Arterial hypertension & $58(87)$ & $64(82)$ & 0.5 \\
\hline Hypercholesterolemia & $49(73)$ & $48(62)$ & 0.16 \\
\hline Diabetes mellitus & $24(36)$ & $28(36)$ & 1 \\
\hline Smoking habit & $11(16)$ & $4(5)$ & 0.03 \\
\hline \multicolumn{4}{|l|}{ Echocardiographic parameters: } \\
\hline Left ventricular ejection fraction, $n(\%)$ & $56(12)$ & $56(14)$ & 0.9 \\
\hline PG-max $[\mathrm{mm} \mathrm{Hg}]$ & $80(26)$ & $98(26)$ & $<0.001$ \\
\hline PG-mean [mm Hg] & $50(20)$ & $59(17)$ & 0.003 \\
\hline \multicolumn{4}{|c|}{ Coexistent coronary and carotid atherosclerosis, $n(\%)$ : } \\
\hline Significant CAD & $42(63)$ & $44(56)$ & 0.5 \\
\hline Relevant ICAS & $11(16)$ & $11(14)$ & 0.8 \\
\hline \multicolumn{4}{|l|}{ Biochemical characteristics: } \\
\hline Total cholesterol [mmol/l] & $4.4(1.1)$ & $4.6(1.2)$ & 0.3 \\
\hline LDL-C [mmol/l] & $2.6(1.0)$ & $2.5(0.9)$ & 0.5 \\
\hline $\mathrm{HDL}-\mathrm{C}[\mathrm{mmol} / \mathrm{l}]$ & $1.4(0.5)$ & $1.3(0.5)$ & 0.3 \\
\hline Triglycerides [mmol/l] & $1.3(0.4)$ & $1.4(0.8)$ & 0.3 \\
\hline eGFR $\left[\mathrm{ml} / \mathrm{min} / 1.73 \mathrm{~m}^{2}\right]$ & 78 (29) & 78 (29) & 0.98 \\
\hline
\end{tabular}

Data are presented as mean (SD) or n (\%). CAD - coronary artery disease, eGFR-estimated glomerular filtration rate, HDL-C-high-density lipoprotein cholesterol, ICAS - internal carotid artery stenosis, LDL-C - low-density lipoprotein cholesterol, PG-max-maximal transvalvular aortic pressure gradient, PG-mean - mean transvalvular aortic pressure gradient. 
$0.8-1.0 \mathrm{~cm}^{2}$ (group A). The two groups did not differ in the proportion of other traditional risk factors or biochemical characteristics. Maximal and mean transaortic pressure gradients were higher in group B, while left ventricle ejection fraction and biochemical characteristics were comparable in both groups. The proportion of subjects with coexistent significant CAD or relevant ICAS was similar across the groups (Table I).

Multiple logistic regression confirmed an independent association of stenosis severity with female gender, while a weak positive effect of age did not reach statistical significance (Table II). Curiously, stenosis severity was not more pronounced with increasing number of classical atherosclerotic risk factors; on the contrary, an opposite, yet not significant, relationship was observed (Table II).

There was no interaction between age and gender in terms of the categorized degree of stenosis severity ( $p=$ $0.3)$. In other words, the proportion of AS patients with an AVA $<0.8 \mathrm{~cm}^{2}$ was higher in those with an over-median age ( $>76$ years) compared to their younger counterparts both in women $(31 / 42$ vs. $12 / 24$, i.e. $74 \%$ vs. $50 \%$, $p=0.06)$ and men $(14 / 28$ vs. $21 / 51$, i.e. $50 \%$ vs. $41 \%$, $p=0.5)$.

\section{Discussion}

Our principal finding was a lack of differences in the prevalence of traditional atherosclerotic risk factors according to the degree of AS. Such a difference might be expected assuming a putative contribution of atherosclerotic risk factors to the development of degenerative AS $[8,9]$, keeping in mind the progressive pattern of AS. Our multivariate regression analysis showed a lower, albeit insignificantly, number of classical risk factors in patients with more severe AS.

However, our results are consistent with a study by Ortlepp et al. [10], who reported that the presence of coexistent $C A D$, but not severe calcific AS by itself, was associated with increased prevalence of traditional risk factors. In their study, none of the traditional risk factors, including hypertension, hypercholesterolemia, diabetes mellitus and smoking, showed a significantly increased proportion in patients with severe AS compared to those with a normal aortic valve, irrespective of the presence of significant CAD on angiography. In particular, Ortlepp et al. [10] described a lower prevalence of smoking habit in AS patients compared to non-matched controls without AS, in some analogy to a decreased percentage of smokers in subjects with more severe AS in the present study. Additionally, in that study, CAD prevalence increased gradually with the increasing number of risk factors [10], in agreement with our previous analysis focused on determinants of the presence of coexistent atherosclerosis in the same study group [7]. Accordingly, since in the present study the prevalence of coexistent significant CAD or relevant ICAS was similar in those with
Table II. Logistic regression analysis of predictors of an aortic valve area below $0.8 \mathrm{~cm}^{2}$

\begin{tabular}{lcc} 
Predictor variable & \multicolumn{2}{c}{ Odds ratio (OR) } \\
\cline { 2 - 3 } & Mean OR (95\% Cl) & $P$-value \\
\hline Age (per 10-year increment) & $1.47(0.97-2.23)$ & 0.07 \\
\hline Gender (women vs. men) & $2.23(1.03-4.81)$ & 0.04 \\
\hline $\begin{array}{l}\text { Number of risk factors } \\
\text { (per increment of 1) }\end{array}$ & $0.71(0.47-1.06)$ & 0.09 \\
\hline eGFR & & 0.84
\end{tabular}

(per rise of $10 \mathrm{ml} / \mathrm{min} / 1.73 \mathrm{~m}^{2}$ )

$\mathrm{Cl}$ - confidence interval, eGFR - estimated glomerular filtration rate. $p=0.3$ by the goodness-of-fit Hosmer-Lemeshow test for the regression equation.

an AVA $<0.8 \mathrm{~cm}^{2}$ and $0.8-1.0 \mathrm{~cm}^{2}$, this is likely to explain comparable percentages of the traditional risk factors in groups $\mathrm{A}$ and $\mathrm{B}$.

Admittedly, we did not study control subjects without severe AS. Nevertheless, similar mechanisms appear to account for the development of severe AS and its further progression. Therefore, our results support the notion that the link between AS and atherosclerotic risk factors may be mainly due to their association with accompanying CAD [10]. In line with this concept, statins failed to retard the progression of degenerative $A S$ in a recent meta-analysis [11]. So, despite some similarities, atherosclerosis and degenerative AS may be regulated by different pathways, which was also suggested by a lack of correlation between aortic valve calcification and coronary artery calcium score or ascending aorta calcifications in patients aged $\geq 60$ years [12]. Notably, only age and male gender, but not other risk factors, were significant independent predictors of progression to AS over a mean 5-year follow-up in elderly subjects with normal aortic valves or aortic valve sclerosis without AS at baseline [13]. Furthermore, after exclusion of patients with CAD, traditional risk factors were not associated with subsequent surgery for AS over a mean follow-up of 10.5 years in a recent nested case-referent prospective study [14], which also supports our findings.

That our study patients with more advanced valve stenosis were older by about 5 years is consistent with progression of degenerative AS with age. Additionally, a higher proportion of females in this subgroup is not unexpected, keeping in mind the 8-year longer average life expectancy in women compared to men in Poland [15].

In a previous analysis of the same study group [7], we proposed a "survival bias" to explain an observation that the prevalence of significant coronary atherosclerosis in AS patients with an over-median age ( $>76$ years) was significantly higher compared to their younger counterparts only in men ( $89 \%$ vs. $55 \%$, respectively, $p=0.002$ ), while the corresponding proportions were similar in women, exhibiting, counterintuitively, even a slight opposite tendency (48\% vs. 54\%) [7]. The hypothetical survival bias 
could result from a high mortality in women with severe AS and coexistent CAD, which might have abolished the expected age-dependent rise in the prevalence of significant CAD in women with severe AS [7].

In contrast, in the present analysis, a higher prevalence of more advanced AS in older AS patients (aged $>76$ years) compared to younger AS subjects was observed irrespective of sex, i.e. both in men (50\% vs. $41 \%$ ) and women ( $74 \%$ vs. $50 \%$ ). This finding strengthens the previously proposed concept of survival bias, being concordant with the notion that a hypothetically altered mortality pattern in women with severe AS could be linked to coexistent CAD. Accordingly, we had previously found an interaction between age and gender with regard to concomitant prevalence of significant coronary atherosclerosis [7]. However, such an interaction was absent in the present study regarding the degree of AS severity. The latter would be expected if that interaction were related to a higher premature mortality of women owing to more severe AS, but not to concomitant CAD.

\section{Conclusions}

Within patients with severe AS, the degree of stenosis severity is associated with a similar prevalence of coexistent coronary and carotid atherosclerosis and atherosclerotic risk factors. Thus, a link between AS and risk factors may result from their association with concomitant atherosclerosis rather than AS by itself.

\section{Conflict of interest}

The authors declare no conflict of interest.

\section{References}

1. Passik CS, Ackermann DM, Pluth JR, et al. Temporal changes in the causes of aortic stenosis: a surgical pathological study of 646 cases. Mayo Clin Proc 1987; 62: 119-23.

2. Dare AJ, Veinot JP, Edwards WD, et al. New observations on the etiology of aortic valve disease: a surgical pathologic study of 236 cases from 1990. Hum Pathol 1993; 24: 1330-8.

3. lung B, Baron G, Butchart EG, et al. A prospective survey of patients with valvular heart disease in Europe: The Euro Heart Survey on Valvular Heart Disease. Eur Heart J 2003; 24: 1231-43.

4. Iung B, Vahanian A. Valvular heart disease in elderly people. Lancet 2006; 368: 969-71.

5. Vahanian A, Alfieri O, Andreotti F, et al.; The Joint Task Force on the Management of Valvular Heart Disease of the European Society of Cardiology (ESC) and the European Association for Cardio-Thoracic Surgery (EACTS). Guidelines on the management of valvular heart disease (version 2012). Eur Heart J 2012; 33: 2451-96.

6. Minners J, Allgeier M, Gohlke-Baerwolf C, et al. Inconsistent grading of aortic valve stenosis by current guidelines: haemodynamic studies in patients with apparently normal left ventricular function. Heart 2010; 96: 1463-8.

7. Bobrowska B, Zasada W, Surdacki A, et al. Predictors of coronary and carotid atherosclerosis in patients with severe degenerative aortic stenosis. Int J Med Sci 2013; 10: 1361-6.
8. Aronow WS, Schwartz KS, Koenigsberg M. Correlation of serum lipids, calcium, and phosphorus, diabetes mellitus and history of systemic hypertension with presence or absence of calcified or thickened aortic cusps or root in elderly patients. Am J Cardiol 1987; 59: 998-9.

9. Stewart BF, Siscovick D, Lind BK, et al. Clinical factors associated with calcific aortic valve disease. J Am Coll Cardiol 1997; 29: 630-4.

10. Ortlepp JR, Schmitz F, Bozoglu T, et al. Cardiovascular risk factors in patients with aortic stenosis predict prevalence of coronary artery disease but not of aortic stenosis: an angiographic pair matched case-control study. Heart 2003; 89: 1019-22.

11. Zhao Y, Nicoll R, He YH, et al. The effect of statins on valve function and calcification in aortic stenosis: a meta-analysis. Atherosclerosis 2016; 246: 318-24.

12. Galas A, Hryniewiecki T, Michałowska I, et al. Aortic valve calcification in 499 consecutive patients referred for computed tomography. Arch Med Sci 2015; 11: 952-7.

13. Novaro GM, Katz R, Aviles RJ, et al. Clinical factors, but not C-reactive protein, predict progression of calcific aortic-valve disease: the Cardiovascular Health Study. J Am Coll Cardiol 2007; 50: 1992-8.

14. Ljungberg J, Johansson B, Engström KG, et al. Traditional cardiovascular risk factors and their relation to future surgery for valvular heart disease or ascending aortic disease: a case-referent study. J Am Heart Assoc 2017; 6: e005133.

15. Central Statistical Office of Poland. Available at: http://stat.gov. $\mathrm{pl} / \mathrm{en} /$ topics/population/life-expectancy/. Accessed August 23, 2017. 